\title{
Synaptic dynamics in complex self-assembled nanoparticle networks
}

\author{
S. K. Bose, ${ }^{a}$ S. Shirai, ${ }^{a}$ J. B. Mallinson, ${ }^{a}$ and S. A. Brown ${ }^{a}$
}

December 27, 2018

\begin{abstract}
We report a detailed study of neuromorphic switching behaviour in inherently complex percolating networks of self-assembled metal nanoparticles. We show that variation of the strength and duration of the electric field applied to this network of synapse-like atomic switches allows us to control the switching dynamics. Switching is observed for voltages above a well-defined threshold, with higher voltages leading to increased switching rates. We demonstrate two behavioral archetypes and show how the switching dynamics change as a function of duration and amplitude of the voltage stimulus. We show that the state of each synapse can influence the activity of the other synapses, leading to complex switching dynamics. We further demonstrate the influence of the morphology of the network on the measured device properties, and the constraints imposed by the overall network conductance. The correlated switching dynamics, device stability over long periods, and the simplicity of the device fabrication provide an attractive pathway to practical implementation of on-chip neuromorphic computing.
\end{abstract}

\section{Introduction}

The quest to replicate the most advanced computer, i.e. the biological brain, has led to immense advancements in software and hardware emulation of the organic neuronal and synaptic structures. $1+3$ The central concepts are that nanoscale switching elements, whose state (resistance) reflects the history of their inputs (hence the name 'memristors') ${ }^{4}$ can be used to emulate the functions of biological synapses, and that networks of such elements could potentially replicate some of the functions of the brain. ${ }^{5-11]}$ Recent work has shown that memristor networks can potentially be used for many tasks, ranging from simple pattern recognition and associative learning $\frac{12 \mid 13}{10}$ more complex applications requiring greater functionality. $6-8 \mid 11$

A wide variety of types of memristive element ${ }^{14-16}$ and types of network ${ }^{9-11}$ have been proposed, each of which has strengths and weaknesses. Highly organised arrays of memristors ${ }^{7 / 17}$ (18 can take advantage of established lithography techniques, but suffer from the same cost and

${ }^{0 a}$ The MacDiarmid Institute for Advanced Materials and Nanotechnology, School of Physical and Chemical Sciences, University of Canterbury, Private Bag 4800, Christchurch 8140, New Zealand. E-mail: simon.brown@canterbury.ac.nz; saurabh.bose@canterbury.ac.nz

This is a post-peer-review, pre-copyedit version of an article published in Faraday Discussions. The final authenticated version is available online at: http://dx.doi.org/10.1039/c8fd00109j 

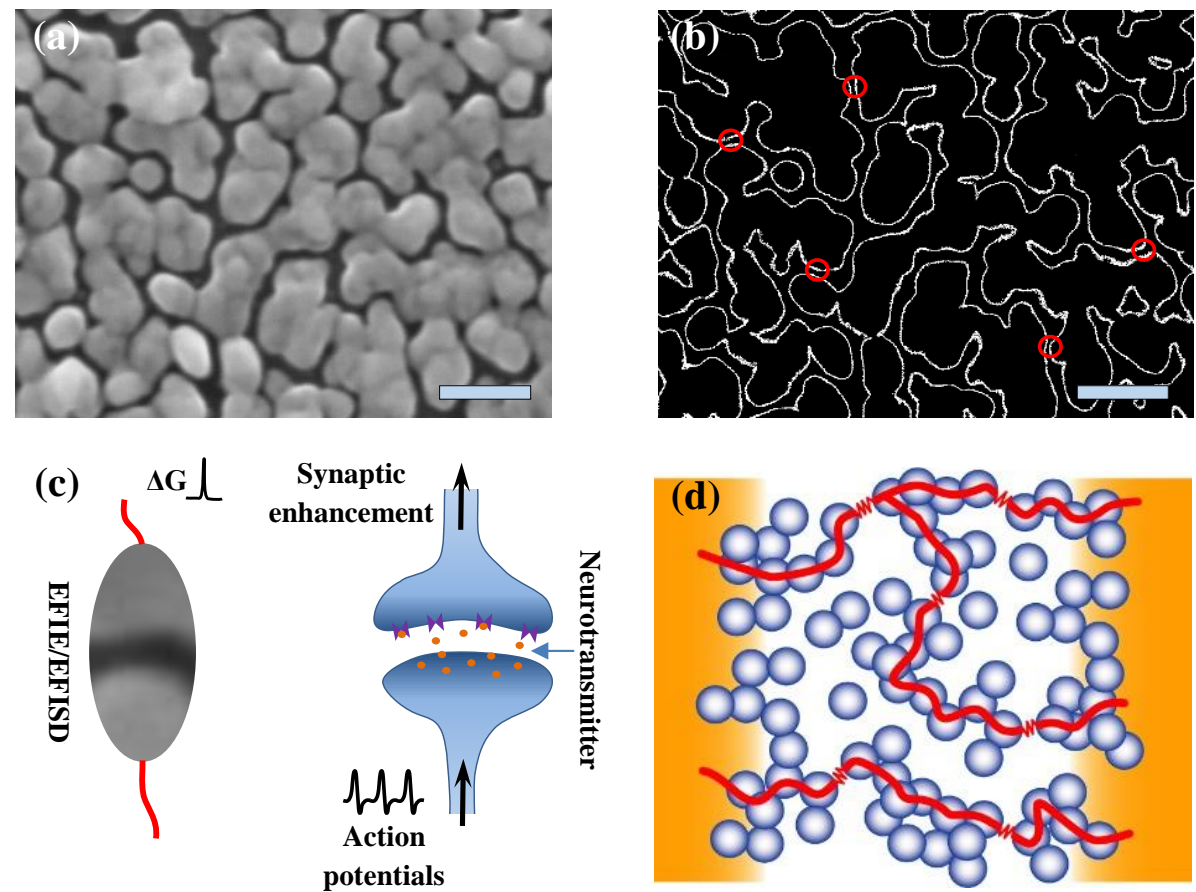

Figure 1 (a) Scanning electron micrograph of a typical part of the nanoparticle network poised near percolation threshold. (b) Corresponding edge-marking emphasizing the percolating nature of the conduction paths along with the spatially distributed tunnel gaps, which are potential atomic-wire formation sites. Some are highlighted by red circles. The scale bars are $100 \mathrm{~nm}$. (c) On receiving requisite action-potential a biological synapse has a finite probability for enhanced synaptic connection. Similarly voltage-stimulus on individual atomic-switch synapses results in atomic movements in the tunnel gap via electric-field-induced-evaporation (EFIE)/ electric-field-induced-surface-diffusion (EFISD) processes. There is a finite probability of atomic-wire formation in the tunnel-gap with an associated increase in conductance (measured as $\Delta \mathrm{G}$ ). (d) Such synaptic reconfigurations of atomic-wires results in dynamical activity involving multitudes of interconnected signal pathways. Only a few representative pathways between the electrodes are depicted here.

fabrication limitations as traditional complementary metal-oxide-semiconductor (CMOS) devices. ${ }^{1920}$ Self-organised networks ${ }^{10121}$ might provide similar functionality using low-cost fabrication techniques as well as genuinely neuromorphic structures that have the built-in complexity that is believed to be important for brain-like functionality. ${ }^{22 \mid 23}$

Here we focus on networks of metallic nanoparticles which, when poised near the percolation threshold, exhibit complex switching behaviour due to the formation and annihilation of atomic scale wires within tunnel gaps in the network ${ }^{24}$. Switching events that cause increases (decreases) in device conductance $G_{\uparrow}\left(G_{\downarrow}\right)$ are understood ${ }^{24 \mid 25}$ to be due to electric-field-induced formation $^{26}$ (electromigration-induced annihilation ${ }^{27}$ ) of atomic-wires in tunnel gaps within the network. These atomic-switches act as inorganic-synapses and multiple such switching events (synaptic reconfigurations) in different locations lead to complex switching dynamics. It has recently been shown that these synaptic networks can be fabricated such that devices work reproducibly for several months. $\stackrel{28}{2}$

Since the networks are complex, it is intrinsically difficult to separate out the switching of 
the individual elements from the observed changes of the network as a whole. Here we apply controlled voltage pulses and characterize the resultant network dynamics. We show that there is a well-defined threshold voltage $\left(V_{T}\right)$ for the onset of switching, and that the atomic wire formation processes occur effectively instantaneously on the timescale of our existing measurement system. Synaptic reconfiguration with voltage stimulus close to $V_{T}$, where wire breaking processes occur on longer time scales and individual switching events can be resolved, simplifies the data analysis. The formation and annihilation of atomic-wires in individual synapses appear to be temporally stochastic, which is believed to be advantageous for some neuromorphic computation paradigms ${ }^{1629}$. Each synapse is inter-connected with the complex network and we show here preliminary evidence suggesting heavy tailed distributions of inter-event-intervals (times between switching events), which are characteristic of correlations. ${ }^{930}$ We also show that the morphology of the network controls its resistance and therefore shifts the measured conductance away from the quantised conductance values expected for individual atomic scale wires. 2431 Finally we outline the prospects for further device and applications development.

\section{Experimental}

Our devices are fabricated using simple cluster deposition techniques that have been described in detail previously ${ }^{28132}$. Briefly, tin clusters $(\sim 8.5 \mathrm{~nm})$ are deposited between Au contacts (100 $\mu \mathrm{m}$ separation) in a controlled atmosphere containing a small amount of air $\left(P_{\text {dep }} \sim 1 \times 10^{-5}\right.$ Torr) with high relative humidity, ${ }^{28}$ resulting in the morphology shown in Fig. 1 (a). Deposition is terminated when the device resistance reaches $\sim 2 \mathrm{k} \Omega\left(G \sim 6 G_{0}\right.$, where $G_{0}=2 e^{2} / h$ is the quantum of conduction ${ }^{33134}$ ).

\section{Results I: Network dynamics}

\subsection{Synaptic network of atomic-switches}

Near the percolation threshold, networks of self-assembled nanoparticles contain a multitude of interconnected atomic-switches. ${ }^{212428}$ Fig. 11 (a) shows a typical scanning electron micrograph (SEM) of such a network with percolating pathways. The corresponding edge marking in Fig. 1 (b) reveals the presence of gaps in these pathways, some of which are indicated by red-circles. These are potential zones for atomic-wire formation (and annihilation) which can be modelled ${ }^{14}$ as inorganic counterparts of the organic synapses in the biological brain. The arrival of an appropriate action potential on a biological synapse has a finite probability of enhancing the synaptic connection in Fig. 1(c). In our inorganic-synapse (atomic-switch), the voltage-pulse stimulus can initiate electric-field induced atomic-rearrangements leading to formation of an atomic-wire bridging the gap, and thus enhancing the overall device conductance. Continuous current flow through these wires can reset the synapse via electromigration. The effect of individual synaptic reconfigurations on the measured device conductance i.e. $G_{\uparrow}\left(G_{\downarrow}\right)$ is dependent upon the spatial as well as temporal configuration, i.e. the position of the synapse in the network. A very simplistic depiction of such a network is shown in Fig. 1 (d). This complex synaptic network could be imagined as a dynamic reservoir ${ }^{12 / 35 / 36}$ of interconnected and interdependent synaptic 'weights'. Reservoir computing (RC) using such atomic switch networks is believed to be a powerful tool for a range of problems including pattern recognition $\underline{10 \mid 28 / 35 / 37}$ and various waveform regression tasks 38 . 

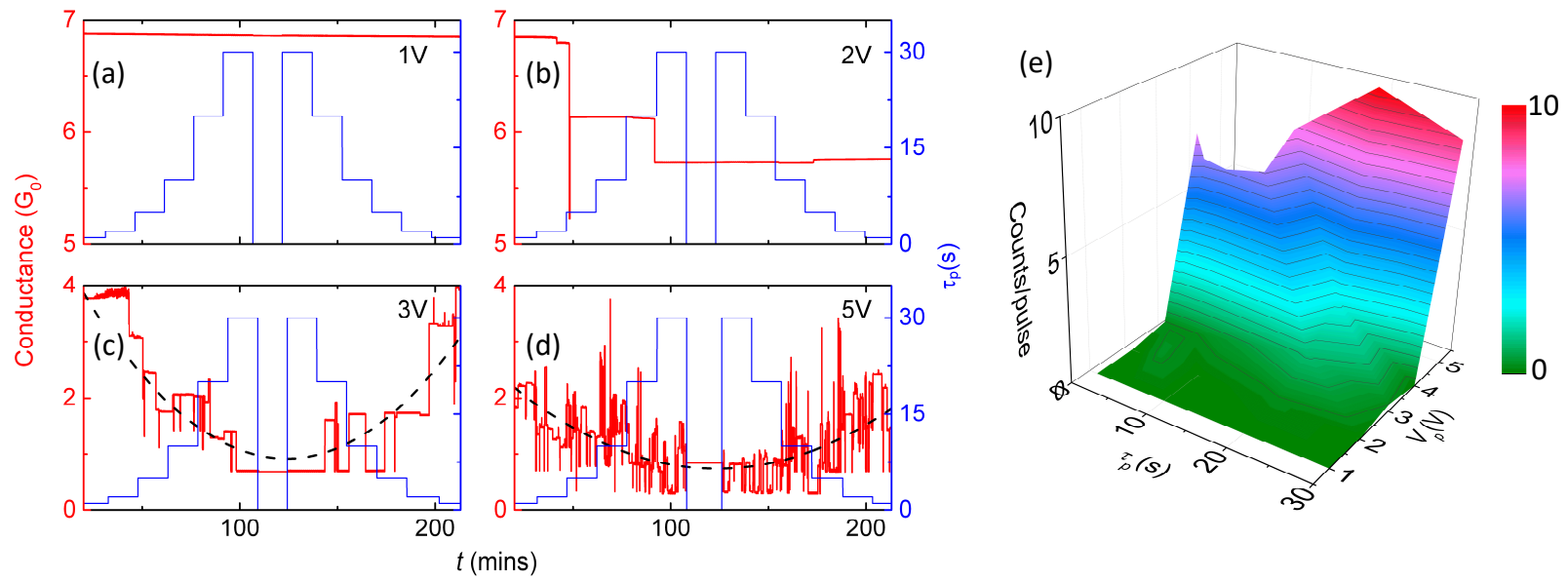

Figure 2 Pulse width $\tau_{p}$ and voltage $V_{p}$ dependence of the switching behaviour. (a - d) Standardized pulse sequence ( $\tau_{p}$ from 2 - 30s) with $V_{p}=1,2,3$ and $5 \mathrm{~V}$. (e) Summary plot of the switching rate as a function of both $\tau_{p}$ and voltage $V_{p}$. Note the dramatic increase in switching rate with increasing voltage.

\subsection{Network activity and event-rates}

Excitation of the complex network with voltage pulses initiates synaptic reconfigurations as shown in Fig. 2. The variation of conductance of a typical device in response to our standard pulse sequence (pulse widths $\tau_{p}$ from $2 \mathrm{~s}$ to 30 s and back to $2 \mathrm{~s}$ ) and when the pulse amplitude $V_{p}$ is increased is shown in Figs. 2 (a) to (d). The $1 \mathrm{~V}$ and $2 \mathrm{~V}$ pulses result in few switching events, whereas voltages above $V_{T} \sim 3 \mathrm{~V}$ lead to a characteristic dependence on pulse width (parabolae are guides to the eye) in which the long pulses result in a lower average $G$. There are absolutely no switching events for read voltages [ 100mV, centre regions in Fig. 2(a-d)]: this stability for sub-threshold voltages $\left(V_{p}<V_{T}\right)$ has been verified for extended periods (weeks) of time. ${ }^{28}$ Every time a synapse connects/disconnects the electric field and current distribution is rearranged and subsequent synaptic activity is dependent upon the modified network state. As $V_{p}$ is increased beyond $V_{T}$ switching becomes more active and the devices reach a state in which the average conductance is $\sim 2 G_{0}$. Active switching around this conductance value can be observed for many months. ${ }^{28}$ Fig. 2(e) summarizes the dependence of the number of events/pulse as a function of $\tau_{p}$ and $V_{p}$, and emphasises the dramatic increase in activity of the network for stronger excitation pulses (higher $V_{p}$ ).

This enhanced device activity (higher rate of events) could be due to either increased activity of the same synapses or activation of new previously inactive synapses. To better understand this, results from numerical simulations ${ }^{21}$ are shown in Fig. 3. Similar to experimental measurements, the voltage stimulus is applied on the left side of the network with right side being grounded. Various groups of ohmically connected particles are connected via the active synapses indicated by green markers (i.e. atomic-switches that reconfigured). As can be seen in Fig. 3(b) the overall density of the active synapses increases at higher voltage which suggests that the experimental observation of increased event-rates at higher voltages can be attributed, at least partially, to the activation of additional synapses. This can be understood intuitively as being due to the increased electric field in tunnel junctions in which the field was previously below a minimum value required to cause atomic motion. In the next section we explore the synaptic dynamics using temperature dependent measurements. 

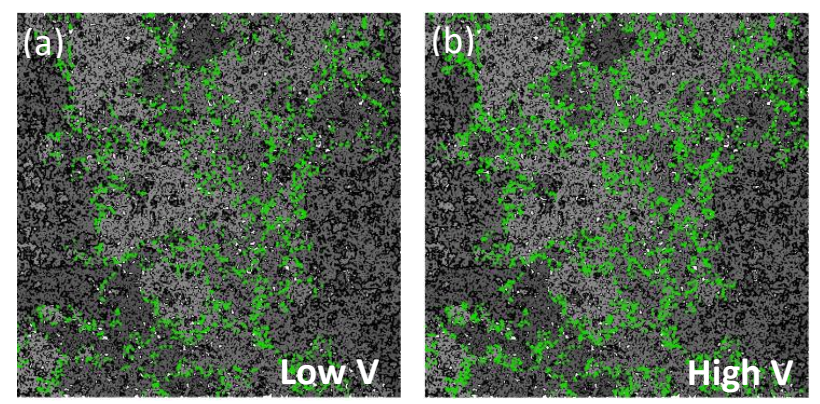

Figure 3 Numerical simulation of network activity in a $200 \times 200$ network (measured in units of particle size) at low and high voltages. The green markers denote the atomic-switches that reconfigured (active synapses) between the gray shaded ohmically connected groups of nanoparticles. The details about the simulations are discussed elsewhere 21 . The increased density as well as spatial distribution of the active synapses at higher voltages (b) indicates activation of previously inactive synapses when lowvoltage was applied (a).

\subsubsection{Temperature dependence of event rates}

The synaptic activity can be arrested by decreasing the device temperature $(T)$. As shown in Fig. 4 lowering the temperature below $T_{t h} \sim 200 \mathrm{~K}$, results in no switching events being observed even when $8 \mathrm{~V}$ pulses are applied. Application of different voltage pulse amplitudes and pulsewidths (not shown here) leads to similar results. This demonstrates that the typical energy scale necessary for atomic-reconfiguration is $\sim 17 \mathrm{meV}$. This energy scale is very similar to the Debye temperature of $\mathrm{Sn}^{39}$. Below $200 \mathrm{~K}$ the atomic motion is 'frozen out', effectively preventing diffusion of adatoms in the tunnel gaps and eliminating the atomic-wire formation processes.

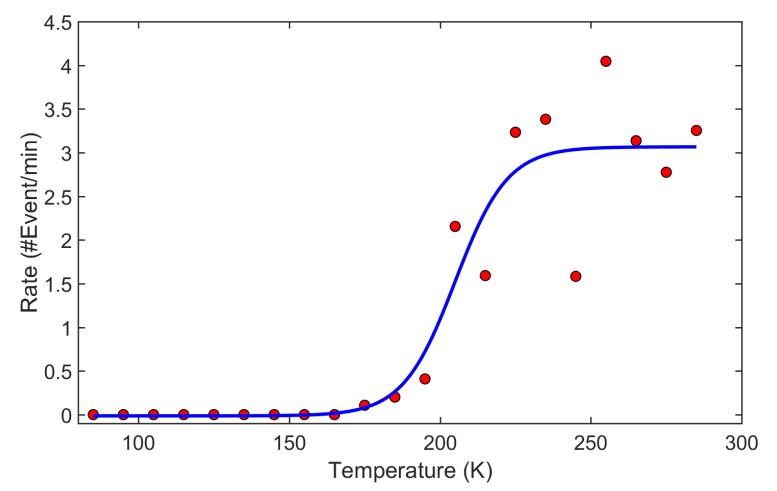

Figure 4 Typical temperature variation of the switching activity, with $8 \mathrm{~V}$ pulses $\left(\tau_{p}=10 s\right)$, shows that there is no switching activity below T $200 \mathrm{~K}$. Switching is activated around $200 \mathrm{~K}$ and the event rates saturate after $250 \mathrm{~K}$. The event rates are averaged every $10 \mathrm{~K}$ and the solid line is sigmoid-fit.

\subsection{Threshold switching: archetype-A}

We now explore the switching dynamics in the near threshold regime $\left(\mathrm{V} \sim \mathrm{V}_{t h}\right)$ where the number of synaptic events is minimized and thus the switching behaviour simplifies. Any voltage pulse stimulus consists of four components i.e. a leading-edge (LE) where voltage increases from off (read voltage $0.1 \mathrm{~V}$ ) to on, trailing-edge (TE) when V decreases from on to off, and on and off regions. Fig. 5(a) presents a typical snapshot example of a G(t) trace with $V_{p}$ close to but above $V_{T}$. The green markers label events at the pulse-edges as "linked-events" and the red 

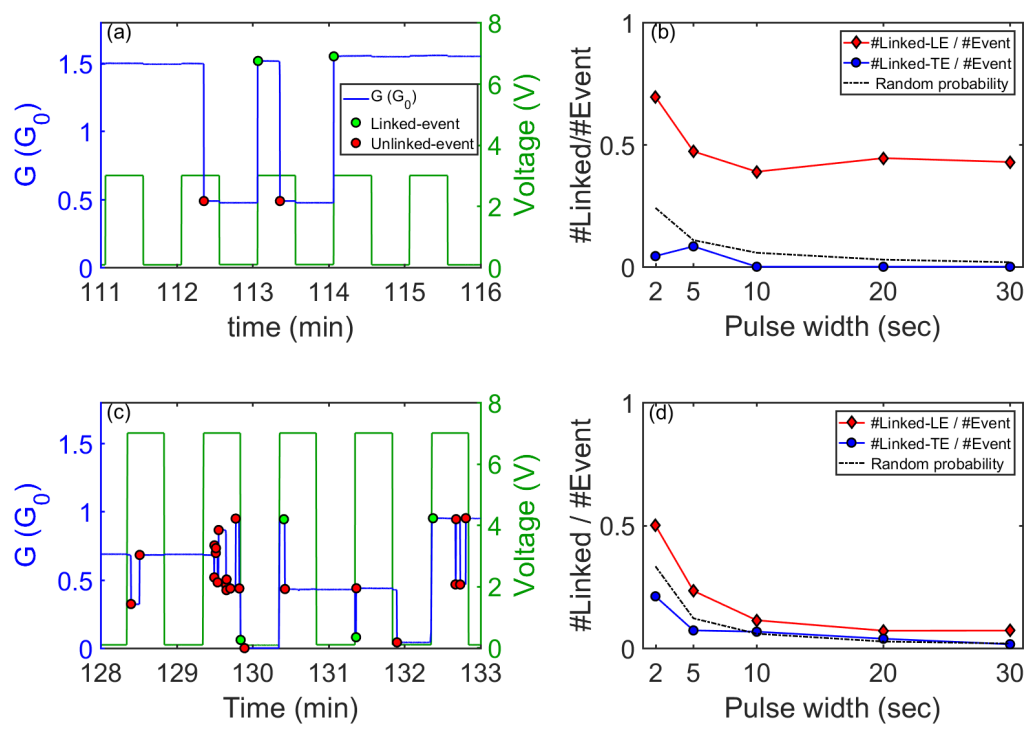

Figure $5(a, c)$ Typical snapshot of $G(t)$ traces depicting the events linked and unlinked with the voltage pulse edges for both archetype-A and $-B$ i.e. at low and high-voltages respectively. The green dots mark the linked-events at pulse-edges and red dots mark unlinked-events. $(b, d)$ The pulse-width dependence of the fraction of linked-events that occur at leading (LE) and trailing (TE) pulse edges. Only the LE linked-events are observed to occur more frequently than would be expected from random statistics. The linked-events at the LE are predominantly up-events, e.g. at $\tau_{p}=30$ s for archetype A, 92\% linkedevents on LE are $\mathrm{G} \uparrow$.

markers denote events in the on region as "unlinked-events". As can be seen in Fig. 5(a) for V $\sim \mathrm{V}_{t h}$ the $G_{\uparrow}$ events occur almost exclusively at the leading edges of the pulses, showing that the electric-field-induced formation of atomic scale wires occurs on a timescale that is effectively instantaneous ${ }^{1}$. In contrast, all $G_{\downarrow}$ events are unlinked, and occur after several seconds of current flow, consistent with the idea that electromigration breaks the atomic scale wires to reset the synapse. We identify this behavior as archetype-A.

Analysis of the switching events over many measurement cycles shows the $G_{\uparrow}$ events are predominantly observed at the leading-edge (LE) of the pulse [Fig. 5(b)]. The fraction of events that are linked at the LE (red-diamonds), as a function of the pulse width $\tau_{p}$, is much higher than possible from a random distribution of events (dotted-line). This indicates that a certain proportion of the tunnel gaps in the network respond instantaneously to the applied electric field. In contrast the number of linked-events at the TE is consistent with random occurrence, i.e. these events occur at the TE simply by chance, they are not driven by the changing electric field.

\section{4 $\mathrm{V}>\mathrm{V}_{t h}$ : archetype-B}

At higher voltages i.e. $\mathrm{V}>V_{t h}$ the archetype-A behavior evolves into more complex archetype-B behavior. Fig. 5(c) depicts a typical G(t) snapshot and shows that, in addition to the linked-up events at LE, additional unlinked events ( $\mathrm{U} \downarrow$ and $\mathrm{U} \uparrow$ ) occur. This behaviour is consistent for all $\tau_{p}$ and across devices. Similar to archetype-A, the linked-events occur at the LE, rather than the TE.

Fig. 6(a) shows the variation of the number of linked-up (L $\uparrow$ ) events at the LE as a fraction of

\footnotetext{
${ }^{1}$ Ref. ${ }^{40 \mid}$ shows that switching can take place on timescales of nanoseconds.
} 

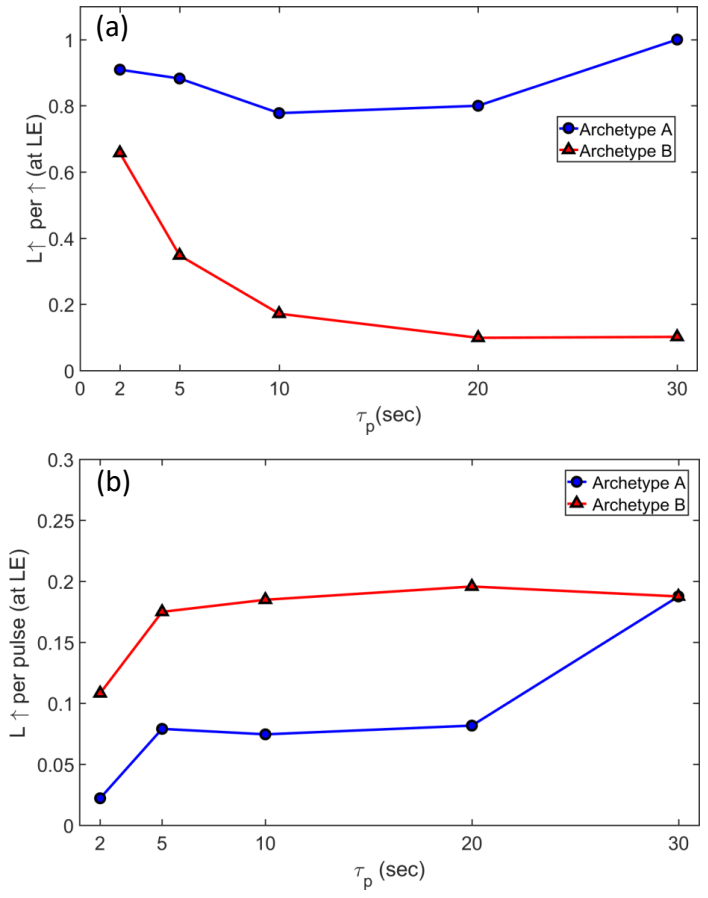

Figure 6 (a) Variation of number of linked-up (L个) events at LE as a fraction of total $\uparrow$ events $(\uparrow=L \uparrow+U \uparrow)$ for both archetypes. In archetype-A, $L \uparrow / \uparrow$ is nearly 1 indicating almost all $\uparrow$ events occur as $L \uparrow$. In archetype-B the $\mathrm{L} \uparrow / \uparrow$ is nearly 0.7 at $\tau_{p}=2$ s and decreases as a function of $\tau_{p}$. This decrease in $\mathrm{L} \uparrow / \uparrow$ for archetype-B is due to additional $U \uparrow$ events (in pairs of $U \uparrow-\bigcup \downarrow$ ) events at higher voltages and longer $\tau_{p}$. (b) The fraction of $L E$ induced $L \uparrow$ events ( $L \uparrow /$ pulse) is in the range around $10-20 \%$ for both archetypes at all $\tau_{p}$ (except low 2s).

the total $\uparrow$ events $(\uparrow=L \uparrow+U \uparrow)$. For archetype-A, $\mathrm{L} \uparrow / \uparrow$ is nearly 1 i.e. $100 \%$, with a negligible fraction of $\mathrm{U} \uparrow$ for all $\tau_{p}$. In contrast, in archetype-B, the ratio $\mathrm{L} \uparrow / \uparrow$ decreases with longer $\tau_{p}$ e.g. at $\tau_{p}=30 \mathrm{~s} \sim 10 \% \uparrow$ events are $\mathrm{L} \uparrow$, with $\sim 90 \%$ being $\mathrm{U} \uparrow$ events. This is because at higher voltages and longer $\tau_{p}$, the increased switching activity results in additional $\mathrm{U} \uparrow$ events. The number of linked-events per pulse on the other hand remains similar for both archetypes (around 10-20\%), as is depicted in Fig. 6(b). Therefore at all voltages, a similar fraction of the LE induce $\mathrm{L} \uparrow$ events.

The event-structure associated with both $\mathrm{L} \uparrow$ and $\mathrm{U} \uparrow$ events across both archetypes is shown in Fig. 7. As can be seen in Fig. 7(a), for archetype-A the L个 events are predominantly (75\%) preceded by a $\mathrm{U} \downarrow$ event. The wide distribution of times $\Delta$ t between $\mathrm{U} \downarrow$ and $\mathrm{L} \uparrow$ events is understood to originate from the distribution of electric-field strengths in the gaps in the percolating network 41 . The electric field induced formation of atomic wire closes the tunnel gap as shown in Fig. 7 of Ref. $\frac{28}{\text {, }}$ on time-scales dependent upon both the electric field strength and the position of the synapse in the network. We believe this is similar to an integrate and fire process. $\frac{42}{42}$

In contrast, for archetype- $\mathrm{B}$ the $\mathrm{U} \uparrow$ events are more common than $\mathrm{L} \uparrow$, and $\mathrm{U} \uparrow$ are also predominantly preceded by a $\mathrm{U} \downarrow$ with a short $\Delta \mathrm{t}$. The narrower distribution of times between the events is due to the higher applied voltage in conjunction with the larger number of active synaptic sites in network. This means that when any one synapse changes state it is likely to cause a change in the state of other synapses, leading to multitude of synapses reconfiguring. Reconfiguration of these interdependent spatially separated synapses via the complex network corresponds to the existence of spatial correlations. 


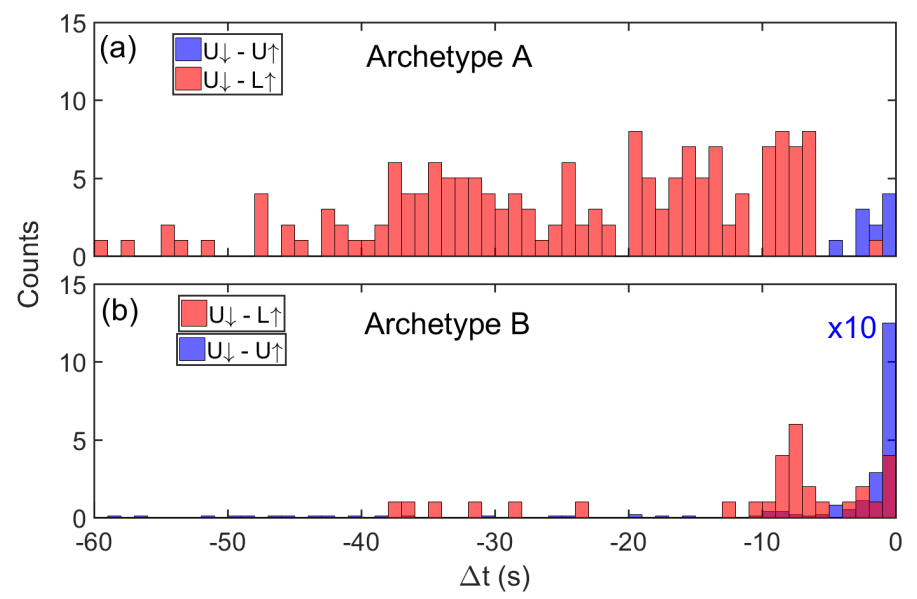

Figure 7 The event-structures associated with linked-up $(\mathrm{L} \uparrow)$ and unlinked-up $(\mathrm{U} \uparrow$ ) events for archetypeA and archetype-B. (a) archetype-A shows predominantly (75\%) of the $L \uparrow$ events are preceded by $U \downarrow$ events with few $U \downarrow-U \uparrow$ pairs. (b) The situation for archetype-B is different with dominance of $U \uparrow$ (note $10 \times$ scale change), typically preceded by an $U \downarrow$. A much smaller number of $L \uparrow$ events are observed, also typically preceded by $\bigcup \downarrow$.

\subsection{Synaptic pathways accessible in archetype A\&B}

As stated in the introduction, all the synaptic elements in the network operate via application of local electric-field (and currents) and are fundamentally similar in character. The relative contribution from individual synapses to the conductance of the network, on the other hand, depends upon their spatial position in the percolating network and thus also determines the network dynamics.

In archetype-A, i.e. at low voltages, the accessible network is limited to a small number of "primary" conduction pathways connecting the two electrodes. The applied voltage drops across gaps in these pathways and generates high local electric fields. Thus a small number of available synapses are able to rapidly form atomic-wires resulting in L $\uparrow$ events at the LE (Fig. 6), with large $\Delta \mathrm{G}$ [see Fig. 10 and associated discussion]. In archetype- $\mathrm{B}$, the synapses on the primary-pathways can still be reconfigured, but the higher voltages also activate additional network-branches. These "higher order" branches make a smaller contribution towards the overall network conductance and synaptic events on them therefore cause a smaller measured $\Delta \mathrm{G}$.

\subsection{Temporal correlations}

To be an effective dynamical reservoir, for example for RC applications, a network must exhibit temporal correlations in addition to the spatial correlations discussed in the previous section. 935|36 Indeed it is believed that spatio-temporal correlations are a feature of the biological brain and are a requirement of neuromorphic network architectures in general.923 Distributions of random, uncorrelated events have statistical distributions of inter-event intervals (IEIs) which decay exponentially. Conversely, correlated non-Poisson processes, where the temporal occurrence of an event depends on the occurrence of previous events, can result in heavy-tailed IEI distributions. 3043

A probability density function (PDF) of IEIs for a typical device is shown in Fig. 8. As previously mentioned, there are no synaptic reconfigurations during the off-pulse and so events with IEIs $\geq \tau_{p}$ are impossible. The raw IEI distribution (red) in Fig. 8 therefore shows strong downward curvature, and at first sight might be mistaken for an exponential decay. However, the 


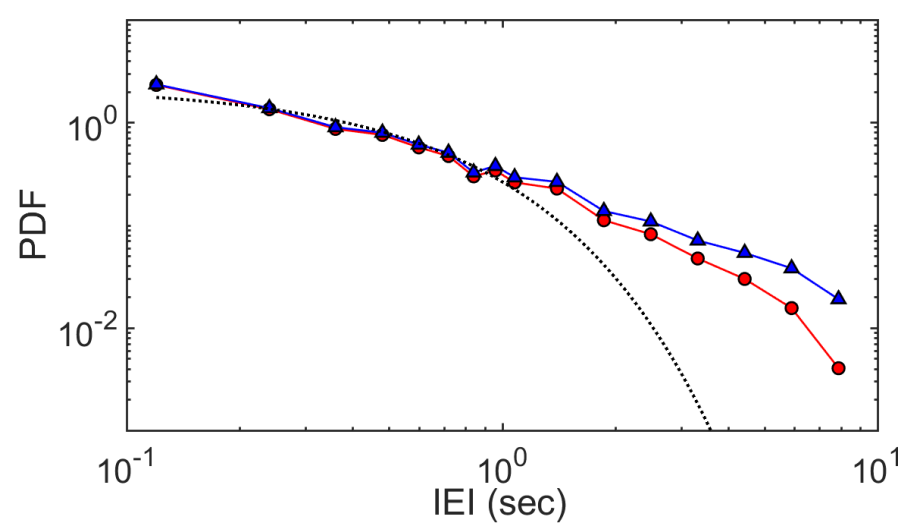

Figure 8 Distribution of inter-event intervals (IEIs) from the device discussed in Figs. 5.7. The raw IEI probability distribution (red circle) shows strong downward curvature at IEI values close to the pulse duration $\left(\tau_{p}=10 \mathrm{~s}, \mathrm{~V}_{p}=8 \mathrm{~V}\right.$ ) due to the effect of the limited pulse length on long-time correlations. In principle, these effects can be corrected for using the correction factor $1 /\left(\tau_{p}-I E I\right)$ which was applied for $\tau_{p}=10 \mathrm{~s}$ (green triangles). Once this effect is removed, the IEI distribution appears to show a heavier-tail, suggesting temporal correlations.

dotted line shows clearly that the best fit exponential does not have a sufficiently long tail to account for the experimental distribution: the tail of the experimental distribution is truncated by the finite pulse length. A straightforward calculation leads to a correction factor of $1 /\left(\tau_{p}-I E I\right)$ that can be applied to remove this effect (blue, $\tau_{p}=10 \mathrm{~s}$ ). Even after this correction, the distribution is still slightly curved, and it is not possible to identify the precise functional form of the data. ${ }^{44}$ Nevertheless the distribution is clearly more heavy-tailed than the exponential distribution, providing preliminary evidence of the temporally correlated network activity. Future experiments will explore the spatio-temporal dynamics in the network.

\section{Results II: Network Morphology}

We now discuss the interdependence of the synaptic activity of individual switches and the network morphology.

\subsection{Interrelationship between individual synapses and the network}

Detailed analysis of archetype-A switching behavior $\left(\mathrm{V}_{p}=3 \mathrm{~V}\right)$ for a different device is shown in Fig. 9, This data is similar to that shown in Fig. 2, and indeed all devices 28 show similar behaviour. In Fig. 9(a), as in Fig. 2(c, d), longer $\tau_{p}$ leads to lower conductance since the electromigration induced disconnection of atomic wires dominates over the field-induced reconnection. Figs. $9(\mathrm{~b})$ and (c) show that histograms of $G$ and $\Delta G$ for each event are strongly peaked. Importantly, the relatively narrow distributions indicate that the individual switching elements accessible in archetype-A exhibit well-defined changes in conductance, consistent with the formation of atomic scale wires ${ }^{24}$ [if the switching elements have a large range of conductances, the resulting changes in conductance of the network must be broadly distributed]. The non-quantised measured values of $\Delta G$ can be explained by the series resistance of the percolating film that surrounds each atomic scale wire - see further discussion in Section 4.2 below.

Fig. 10(a) shows separate histograms of the $\Delta G$ data for representative $\tau_{p}$. $G_{\downarrow}$ events exhibit peaks for $\Delta G \sim-0.3 G_{0}$ whereas $G_{\uparrow}$ events are peaked at $\Delta G \sim 0.3 G_{0}$ only for small pulse widths. For longer pulse widths there are a significant number of large $G_{\uparrow}$ events and the histogram is 

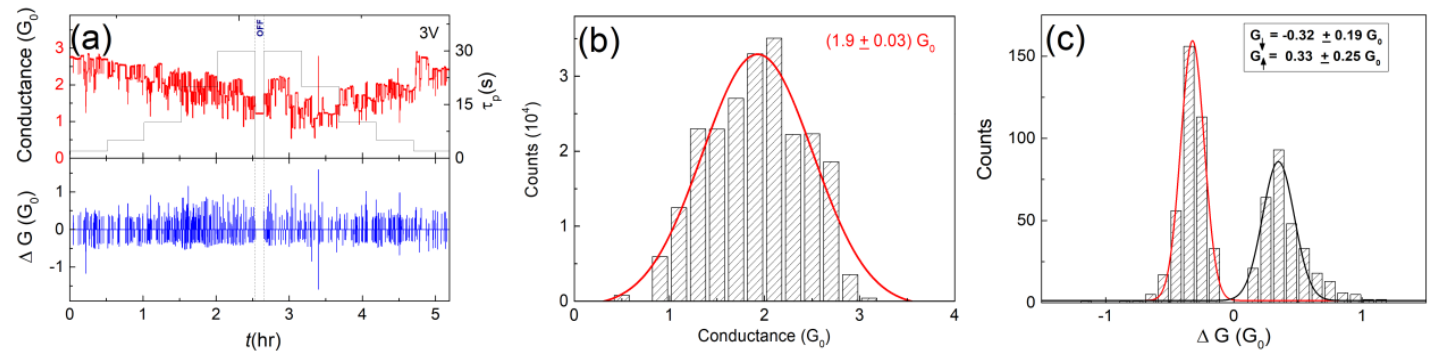

Figure 9 (a) Switching in response to a sequence of square voltage pulses with fixed $V_{p}=3 \mathrm{~V}$ (just above $\left.V_{T}\right)$ and variable $\tau_{p}(2-30 \mathrm{~s})$. The conductance remains unaltered for read voltages of $0.1 \mathrm{~V}$ as seen in the flat section (OFF) in middle of the sequence. Bottom panel shows $\Delta G$ for these switching events. (b) Histogram of the measured initial conductance for each switching event shows a broad peak around $\sim 2 \mathrm{G}_{0}$. (c) Histogram of sizes of switching events indicates a clear preference for $\Delta G= \pm 0.3 G_{0}$. The solid lines in (b) and (c) are Gaussian fits to the distributions.

not strongly peaked. This behaviour can be understood as being the result of the breaking of multiple atomic scale wires during long pulses due to electromigration. This means that when the next pulse is applied there are more synapses available for formation of new wires and larger $\Delta G$ are possible. Fig. 10 (b) shows that $\Delta G$ is not correlated with $G$ for these switching events, consistent with switching events of the same size (i.e. quantised events) taking place at multiple sites in the complex network.

\subsection{Measured conductance changes and device morphology}

In the present devices long-term stability of the switching behaviour was achieved by oxidation during deposition ${ }^{28}$, and so the resistance of the paths through the network is non-negligible. In contrast, in Ref. ${ }^{24}$ the emphasis was on achieving devices that exhibit large switching events (large $\Delta G$ ) and the observation of quantised resistance $\left(G=N G_{0}\right)$, which required a low series resistance. If an atomic scale wire is formed somewhere within a percolating resistive network, the rest of the network can be approximated as an equivalent circuit comprised of a series conductance $\alpha G_{0}$ and a parallel conductance $\beta G_{0}$ [ $\alpha$ and $\beta$ could be any real numbers, whereas $N$ is an integer; all conductances are in units of $\left.G_{0}\right]$. It then requires only a little algebra to show that the total conductance of the network is $G=\alpha \beta /(\alpha+\beta)$ and the change in conductance is $\Delta G=\alpha^{2} N /[(\alpha+\beta)(\alpha+\beta+N)]$.

Fig. 9 shows that, for this particular device and archetype-A behavior near $V_{t h}, \Delta G \sim 1 / 3$ and $G \sim 2$. Although the positions of the peaks in the histograms of $\Delta G$ and $G$ vary to some extent from device to device, such well defined peaks are commonly observed for archetype-A. These peaks are visible because the voltage (corresponding to an electric field in the gaps in the network) is only sufficient to activate a small number of switching sites (synapses). Higher voltages activate more switching sites (Figs. 2(e)) and histograms of $\Delta G$ exhibit power law behaviour as complex inter-dependent pathways through the network are activated - this will be discussed in detail elsewhere.

The results in Fig. 9 put strong constraints on the values of $(\alpha, \beta, N)$ that can provide solutions of the above equations. We find numerically that when $N$ is restricted to physically reasonable ${ }^{\sqrt{45}}$ values less than 10 , the values of $\alpha$ and $\beta$ that meet these constraints are limited to the range $2<\alpha<5.5$ and $\beta<10$. These solutions indicate that in order to achieve the 

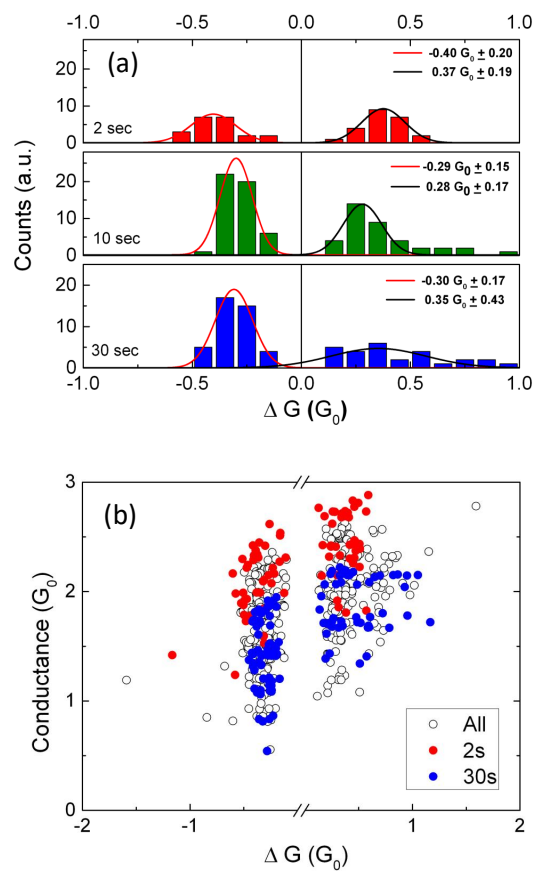

Figure 10 (a) Histograms of switching events for different $\tau_{p}$. For shorter pulse widths the majority of events are centered around $\pm 0.3 G_{0}$. At higher pulse widths $\left(\tau_{p} \sim 20-30 \mathrm{~s}\right)$ the efficient breaking of connections allows an increase in the number of large $G_{\uparrow}$ events. (b) Interdependence of $\Delta G$ and $G$ with open circles representing all events and red and blue filled symbols representing the subset of the data with $\tau_{p}=2 \mathrm{~s}$ and 30 s respectively. The x-axis break indicates that events with $\Delta G>0.1 G_{0}$ are included in the analysis.

experimental results the parallel conductance cannot be too high, and the series conductance is restricted to a relatively narrow range. The range of $\alpha$ corresponds to series resistances $\sim$ 2 - $6 \mathrm{k} \Omega$, while the parallel resistance must be bigger than $\sim 1 \mathrm{k} \Omega$. The measured resistances $(1-10 \mathrm{k} \Omega)$ of the percolating networks that exhibit switching effects are consistent with these ranges.

$\alpha$ and $\beta$ represent the electrical properties of the percolating network and so it follows that the morphology of the actual device must be such that it provides the required series and parallel resistances. The sizes of the nanoparticles and morphology of the network are controlled by coalescence during the deposition process 28 , and in light of this analysis it is clear that one of the functions of the deliberate oxidation process during deposition of the nanoparticles is to constrain the morphology of the network so as to allow observation of consistent switching behaviour.

\section{Discussion and Conclusions}

Our films of nanoparticles exhibit complex patterns of electrical switching behaviour at sites corresponding to tunnel gaps within the percolating network ('synapses'). Voltage pulses were used to control formation and annihilation of atomic scale wires at these sites, thereby modifying the device conductance. Shorter (longer) voltage pulses bias the network towards higher (lower) conductance as more (fewer) connections are formed across the network.

Even in the low $V_{p}$ regime, switching at one site is influenced by the states of the other 


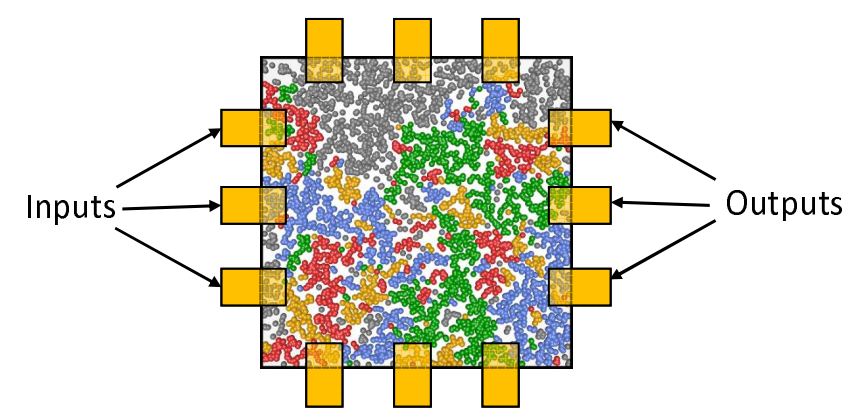

Figure 11 Schematic of the percolating network comprising of many groups of particles separated by tunnelling gaps surrounded by an array of contacts/electrodes which can be designated as input or output terminals depending on the computation task at hand.

synapses. This provides a first indication of the possibility that these percolating networks might exhibit the spatio-temporal correlations required for neuromorphic computing. As the applied voltage is increased, the switching dynamics become more complex because more synapses in the network are activated. We have explored the inter-relationships between the switching events that cause increases and decreases in conductance, and have shown that measured distributions of inter-event intervals are suggestive of the heavy-tailed distributions that are believed to reflect correlated behaviour. 30143

\subsection{Applications}

The atomic scale processes appear to be similar to those that occur in $\mathrm{Ag} / \mathrm{Ag}_{2} \mathrm{~S}$ atomic switches, which allow synaptic learning ${ }^{14}$ and can potentially function on timescales of $<10 \mathrm{~ns} \mathrm{~s}^{40}$. The exploitation of this type of synaptic behaviour in self-organised networks is still in its infancy, but complex dynamics and spatio-temporal correlations are essential for development of neuromorphic applications. Such applications include, for example, spike timing dependent plasticity 14 , learning behaviour ${ }^{8 \mid 1121}$, rate and population coding ${ }^{37}$, detection of temporal correlations ${ }^{16 \mid 29}$ and pattern recognition 5 |912/46. It is clearly important now to explore the ultimate limits of the present switching processes and develop multi-contact devices that would allow demonstration of applications such as pattern recognition.

\subsection{Prospects for further device development}

One of the attractive features of the present networks is the simplicity of the device fabrication process ${ }^{28}$ and the possibility of straightforward extension to a range of more complex devices. Fig. 11] shows a schematic of the percolating network, comprising many groups of particles separated by tunnelling gaps, surrounded by an array of contacts/electrodes which can be designated as input or output terminals. In order to be able to implement (for example) RC we envisage that initially the output signals will be recorded and linear regression tasks performed in software, but that in a future generation of devices the electrodes will provide connections between the percolating network and CMOS circuitry on the same chip, allowing implementation of the required algorithms in hardware. Various other configurations are of course possible where contacts can be used as gate electrodes and used to modulate the percolating network. This could be equivalent to modifying the synaptic weights in the interior of an artificial neural network.

\section{Conflicts of interest}

There are no conflicts to declare. 


\section{Acknowledgements}

The authors gratefully acknowledge financial support from the Ministry of Business Innovation and Employment, New Zealand, the Marsden Fund, New Zealand, and the MacDiarmid Institute for Advanced Materials and Nanotechnology.

\section{Notes and references}

[1] C. Mead, Proc. IEEE, 1990, 78, 1629-1636.

[2] P. A. Merolla and et al., Science, 2014, 345, 668-673.

[3] J. Schmidhuber, Neural Networks, 2015, 61, 85-117.

[4] D. B. Strukov, G. S. Snider, D. R. Stewart and R. S. Williams, Nature, 2008, 453, 80-83.

[5] R. F. Service, Science, 2014, 345, 614-616.

[6] F. Alibart, E. Zamanidoost and D. B. Strukov, Nat. Commun., 2013, 4, 2072.

[7] M. Prezioso, F. Merrikh-Bayat, B. D. Hoskins, G. C. Adam, K. K. Likharev and D. B. Strukov, Nature, 2015, 521, 61-64.

[8] A. Pantazi, S. Woźniak, T. Tuma and E. Eleftheriou, Nanotechnology, 2016, 27, 355205.

[9] A. Z. Stieg, A. V. Avizienis, H. O. Sillin, C. Martin-Olmos, M. Aono and J. K. Gimzewski, Adv. Mater., 2012, 24, 286-293.

[10] A. V. Avizienis, H. O. Sillin, C. Martin-Olmos, H. H. Shieh, M. Aono, A. Z. Stieg and J. K. Gimzewski, PLoS One, 2012, 7, e42772.

[11] A. Serb, J. Bill, A. Khiat, R. Berdan, R. Legenstein and T. Prodromakis, Nat. Commun., 2016, 7, 12611.

[12] M. S. Kulkarni and C. Teuscher, IEEE/ACM Inter- Natl. Symp. Nanoscale Archit., 2012, pp. 226-232.

[13] Y. V. Pershin and M. Di Ventra, Neural Networks, 2010, 23, 881-886.

[14] T. Ohno, T. Hasegawa, T. Tsuruoka, K. Terabe, J. K. Gimzewski and M. Aono, Nat. Mater., 2011, 10, 591-595.

[15] H. Kim, M. P. Sah, C. Yang, T. Roska and L. O. Chua, Proc. IEEE, 2012, 100, 2061-2070.

[16] T. Tuma, A. Pantazi, M. Le Gallo, A. Sebastian and E. Eleftheriou, Nat. Nanotechnol., 2016, 11, 693-699.

[17] Z. Wang, S. Joshi, S. E. Savel'ev, H. Jiang, R. Midya, P. Lin, M. Hu, N. Ge, J. P. Strachan, Z. Li, Q. Wu, M. Barnell, G.-L. Li, H. L. Xin, R. S. Williams, Q. Xia and J. J. Yang, Nat. Mater., 2017, 16, 101-108.

[18] Z. Wang, S. Joshi, S. Savel'ev, W. Song, R. Midya, Y. Li, M. Rao, P. Yan, S. Asapu, Y. Zhuo, H. Jiang, P. Lin, C. Li, J. H. Yoon, N. K. Upadhyay, J. Zhang, M. Hu, J. P. Strachan, M. Barnell, Q. Wu, H. Wu, R. S. Williams, Q. Xia and J. J. Yang, Nat. Electron., 2018, 1, 137-145. 
[19] D. J. Frank, IBM J. Res. Dev., 2002, 46, 235-244.

[20] Y. Taur, D. A. Buchanan, W. Chen, D. J. Frank, K. E. Ismail, L. Shih-Hsien, G. A. Sai-Halasz, R. G. Viswanathan, H. J. C. Wann, S. J. Wind and H. S. Wong, Proc. IEEE, 1997, 85, 486503.

[21] S. Fostner and S. A. Brown, Phys. Rev. E, 2015, 92, 1-11.

[22] D. R. Chialvo, Nat. Phys., 2010, 6, 744-750.

[23] J. M. Beggs and D. Plenz, J. Neurosci., 2003, 23, 11167-11177.

[24] A. Sattar, S. Fostner and S. A. Brown, Phys. Rev. Lett., 2013, 111, 136808.

[25] C. Minnai, A. Bellacicca, S. A. Brown and P. Milani, Sci. Rep., 2017, 7, 7955.

[26] M. Olsen, M. Hummelgård and H. Olin, PLoS One, 2012, 7, e30106.

[27] C. Xiang, J. Y. Kim and R. M. Penner, Nano Lett., 2009, 9, 2133-2138.

[28] S. K. Bose, J. B. Mallinson, R. M. Gazoni and S. A. Brown, IEEE Trans. Electron Devices, 2017, 64, 5194-5201.

[29] D. Querlioz and V. Trauchessec, Proc. - IEEE Int. Symp. Circuits Syst., 2013, pp. 1596-1599.

[30] A.-L. Barabasi and R. Albert, Science, 1999, 286, 509-513.

[31] N. Agraït, A. L. Yeyati and J. M. van Ruitenbeek, Phys. Rep., 2003, 377, 81-279.

[32] J. Schmelzer, S. A. Brown, A. Wurl, M. Hyslop and R. J. Blaikie, Phys. Rev. Lett., 2002, 88, 226802.

[33] D. A. Wharam, T. J. Thornton, R. Newbury, M. Pepper, H. Ahmed, J. E. F. Frost, D. G. Hasko, D. C. Peacock, D. A. Ritchie and G. A. C. Jones, J. Phys. C Solid State Phys., 1988, 21, L209.

[34] B. J. van Wees, H. van Houten, C. W. J. Beenakker, J. G. Williamson, L. P. Kouwenhoven, D. van der Marel and C. T. Foxon, Phys. Rev. Lett., 1988, 60, 848-850.

[35] M. Lukoševičius and H. Jaeger, Comput. Sci. Rev., 2009, 3, 127-149.

[36] H. O. Sillin, R. Aguilera, H.-H. Shieh, A. V. Avizienis, M. Aono, A. Z. Stieg and J. K. Gimzewski, Nanotechnology, 2013, 24, 384004.

[37] A. Mizrahi, T. Hirtzlin, A. Fukushima, H. Kubota, S. Yuasa, J. Grollier and D. Querlioz, Nat. Commun., 2018, 9, 1533.

[38] E. C. Demis, R. Aguilera, K. Scharnhorst, M. Aono, A. Z. Stieg and J. K. Gimzewski, Jpn. J. Appl. Phys., 2016, 55, 1102B2.

[39] G. R. Stewart, Rev. Sci. Instrum., 1983, 54, 1-11.

[40] M. A. K. Terabe, T. Hasegawa, T. Nakayama, Nature, 2005, 433, 47-50. 
[41] S. Fostner, , R. Brown, J. Carr and S. A. Brown, Phys. Rev. B , (2014) Continuum, 2014, 89, 075402.

[42] A. N. Burkitt, Biol Cybern, 2006, 95, 1-19.

[43] M. Karsai, K. Kaski, A.-L. Barabási and J. Kertész, Scientific Reports, 2012, 2, 397.

[44] A. Clauset, C. R. Shalizi and M. E. J. Newman, SIAM Rev., 2009, 51, 661.

[45] L. Olesen, E. Laegsgaard, I. Stensgaard, F. Besenbacher, J. Schiotz, P. Stoltze, K. W. Jacobsen and J. K. Norskov, Phys. Rev. Lett., 1994, 72, 2251-2254.

[46] J. Torrejon, M. Riou, F. A. Araujo, S. Tsunegi, G. Khalsa, D. Querlioz, P. Bortolotti, V. Cros, K. Yakushiji, A. Fukushima, H. Kubota, S. Yuasa, M. D. Stiles and J. Grollier, Nature, 2017, 547, 428-431. 